\title{
The human genome and biomedicine: future goals
}

\section{THE SEQUENCE}

A major accomplishment of the XXI century was celebrated in the month of February 2001 with the publication of the nearly complete sequence of the human genome representing about 3.2 million base pairs by two groups from private (Celera Genomics) and public sectors (International Human Genome Sequencing Consortium) (see Science 291, 1304-135, 2001; Nature 409, 861-921, 2001). The main highlights of the available sequence are the following: there appear to be 30-40,000 protein-coding genes which represents only about twice as many as in the worm or fruit fly; the full set of proteins (the proteome) encoded by the human genome is more complex than those of invertebrates; the genome shows marked variation in the distribution of genes, transposable elements, CpG islands, GC content and recombination rate; hundreds of human genes appear likely to have originated from horizontal transfer form bacteria and dozen of genes appear to have derived form transposable elements; only $1 \%$ of the genome is spanned by exons whereas $24 \%$ is in introns, with 75\% of the genome being intergenic DNA: the pericentromeric and subtelomeric regions of chromosomes are filled with large recent segmental duplication of sequences from elsewhere in the genome; there is strong selection in favour of preferential retention of Alu elements in GC-rich regions and that these «selfish» elements may benefit their human host; the mutation rate is about twice as high in male as in female meiosis; large GC-poor regions are strongly correlated with «dark G-bands» in karyotypes; recombination rates tend to be much higher in distal regions (around 20 megabases) of chromosomes and on shorter chromosome arm in general; more than 1.4 million single nucleotide polymorphisms (SNPs) in the human genome have been identified and less than 1\% of all SNPs resulted in variation of protein; comparative genomic analysis indicates vertebrate expansion of genes associated with neuronal function, with tissue-

Rev Oncología 2001; 3: 119-120 specific developmental regulation, and with the hemostasis and immune systems.

\section{THE NUMBER OF GENES}

An immediate consideration that emerges from the sequence of the human genome is that the number of genes, 31,780 (public sequence) or 39,114 (Celera sequence), is relatively modest compared with other organisms, like the 13,601 genes for the Drosophila melanogaster, the 19,099 genes for the ne$m$ a $\quad t \quad 0 \quad d \quad e$ C. Elegans and the 25,498 genes for the plant Arabidopsis thaliana. This indicates that the encoded human genes must fulfill functions more complex than those of other organisms. Indeed, we know that there are many features in humans clearly distinct from other animal species. However, we share in common many other characteristics. For example, we learned from the nematode that genes that govern cell death (apoptosis) are similarly represented in humans; from yeast, we identified genes that control the cell cycle; from the fruit fly we identified genes that control development. At the structural level, we learned that there are domains in proteins from many species that are conserved in human proteins. Undoubtedly, the use of model systems will be critical in our understanding of the human genome. Celera has nearly sequenced the mouse genome and the information provided will help in deciphering the function of human genes.

\section{THE FUNCTION OF GENES}

Does it mean that having the human genome sequenced we will soon understand the function of all genes in the context of a cell? It is generally agreed that it will take many years before we assemble maps that interrelate the function of most genes. This task is been addressed by the use of the microarray technology that will generate a catalogue of genes and 
their products, primarily messenger RNA transcripts and their cognate proteins. An effort has been initiated by Celera and other groups to identify all of the proteins that are produced in cells (the proteome) using high throughput approaches, like separation of proteins by two-dimensional gels and capillary electrophoresis followed by sequence analysis using mass spectrometry. The purpose will be to identify all of the proteins as they are produced in a cell. Due to protein posttranslational modifications (methylation, glycosilation, phosphorylation, etc) the actual number of proteins within a cell and their functions may be 10 times larger than that predicted from the total number of genes. As we know, protein modifications have important consequences for protein function. Undoubtedly, as the technology for studies of gene expression (transcriptome), metabolism (metabolome), drug action (pharmacogenomics) and protein identification (proteome) together with bioinformatics improvements, we will see in the next few years vast information in these areas of research. This will facilitate studies on the mode of action of any given gene in the context of a defined pathway, i.e signalling, cell grow, differentiation, cell death, immune regulation etc. The advances in mouse genetics, particularly the genetic manipulation that allows the incorporation of a transgene to a specific tissue, the selective inactivation of a gene (knockout), the replacement of the normal gene for a mutant one (knock-in) and the controlled inducible expression of selected genes, will provide critical information to what a gene can do for a cell and for an organism.

\section{THE APPLICABILITY}

How is the information provided by the human genome going to have an immediate use to improve o $u$ u health? A direct application is in diagnosis using the microchip technology in order to identify genes that are activated/inactivated under a pathological condition, like a comparison of the pattern of gene expression between normal and tumor tissue and the identification of mutations in specific genes. Due to genetic variation in the population, it is predicted that a medicine «a la carte» will be implemented per patient. This technology will be introduced in hospitals and carried out either in-house or as an outside service by genomic companies. Another application is in the identification of targets and the development of new drugs. There is a race by the pharmaceutical industry to find new products and targets to control diseases like cancer, infections, cardiovascular, neurological and genetic disorders. The application of functional genomics and proteomics will undoubtedly contribute to the identification of genes and their products that play crucial roles in pathological processes and to subsequently develop drugs with specificity. Recent developments in the application of $\mathrm{NMR}$ and X-ray crystallography to solve the three-dimensional structure of proteins and new programs in bioinformatics to predict structures are paving the way to elucidate many protein structures at a fast rate and to define how drugs might interact with specific amino acid regions. Another immediate utility of the human genome is to facilitate the advancement of knowledge in the research community. While most research groups study the function of individual genes, analyzing the role of a gene within the context of the whole set of genes and then place it in the context of an specific pathway, will provide a closer view of the role of a gene within a cell and in an organism. To carry out this type of research it is necessary to have access to functional genomic services. While industries might eventually provide the chips and their running cost will decline in the next few years it will still be necessary to have the capability to generate chips with a collection of selected genes for functional studies and to be able to process the data. In addition, it will be necessary to continue the sequencing of more model systems and to develop animal models for studies of in vivo function of human genes. An exciting new era has emerged in biomedicine.

Mariano Esteban Centro Nacional de Biotecnología. CSIC 